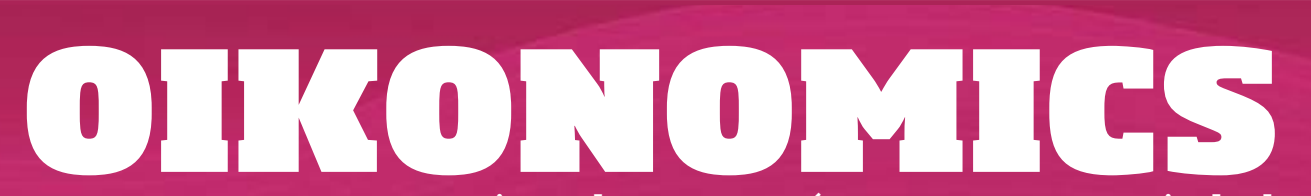

Revista de economía, empresa y sociedad

CAMBIO DE PARADIGMA

\title{
Del yo al nosotros: nuevas formas de liderar
}

\section{Francisco Fermín Mallén Broch}

Universidad Jaume I

\section{Fmilio Domínguez Escrig}

Universidad Jaume I

RESUMEN En los últimos años estamos asistiendo a un cambio de paradigma en las ciencias sociales y en las motivaciones que guían las relaciones entre las personas, desplazándonos desde lo que se ha acuñado como egosystem, donde lo prioritario es el bienestar de uno mismo, a lo que se ha venido a llamar ecosystem, un sistema que prioriza el bienestar de la comunidad. Centrados en el ámbito organizativo, las condiciones del entorno en el que se desenvuelven las empresas, así como acontecimientos recientes relacionados con comportamientos poco éticos y egoístas de sus líderes, están dando paso a un conjunto de estilos de liderazgo, contemporáneos o emergentes, donde los valores humanos adquieren especial relevancia. Numerosos trabajos analizan las consecuencias para el individuo, el grupo y la organización de esta nueva corriente de estilos de liderazgo, pero otros aconsejan el análisis de las consecuencias de comportamientos o conductas concretas de los líderes: comportamiento altruista, humildad, empoderamiento, capacidad de perdonar y orientación hacia la sostenibilidad, por citar algunos ejemplos. Se presentan algunas conclusiones y retos de esta nueva línea de trabajo.

PALABRAS CLAVE liderazgo; liderazgo servicial; liderazgo ético; altruismo; empoderamiento 


\title{
From me to us: new ways to lead
}

\begin{abstract}
In recent years, we have witnessed a paradigm shift in social sciences and the motivations that steer the relationships between people, moving from what has been described as an 'ego-system', in which priority is given to one's own wellbeing, to what could be referred to as an 'ecosystem', in which the wellbeing of the community is prioritized. Focusing on the organizational sphere, the conditions of the environment in which companies operate, as well as recent events related to the unethical and selfish conduct of their leaders, are paving the way for a new set of contemporary or emerging leadership styles in which human values are attributed particular significance. A number of studies analyse the consequences of this new trend of leadership styles for individuals, groups and organizations, but other studies recommend an analysis of the consequences of the specific behaviours or conducts of the leaders: altruism, humility, empowerment, capacity for forgiveness and focus on sustainability, to give just a few examples. Some conclusions are presented, as well as the challenges posed in this new line of work.
\end{abstract}

KEYWORDS leadership; servant leadership; ethical leadership; altruism; empowerment

\section{Introducción}

Al estudiar el rol que los líderes desempeñan en las organizaciones, no se pueden pasar por alto las condiciones del entorno en el que estas desarrollan su actividad, haciendo frente a cambios cada vez más frecuentes e inesperados. Las características del nuevo contexto competitivo están modificando la manera de trabajar en las empresas, lo que demanda nuevos estilos de dirección que se alejen de los clásicos estilos transaccionales (Zhu y otros, 2005), basados en premios y castigos para conseguir lo que se desea de los empleados. En una economía basada en el conocimiento, la competitividad de las empresas depende cada vez más de las personas y esta tendencia exige tener en cuenta sus necesidades más que nunca.

Además, algunos acontecimientos y noticias recientes han puesto en tela de juicio la conducta de algunos líderes y todo ello ha generado la necesidad de nuevos puntos de vista y formas de actuar por su parte. El abuso de poder, las emociones tóxicas, el aislamiento social en el puesto de trabajo y otras acciones limitan no solamente el bienestar psicológico de los empleados y su conciliación de la vida 
laboral y personal (De Cieri y otros, 2005), sino también el bienestar de la sociedad en general.

Este tipo de problemas está presente a nivel mundial, con casos tan conocidos como los que han llevado a la desaparición de Enron, WorldCom y Tyco en Estados Unidos, y casos más cercanos, algunos de ellos protagonizados por entidades financieras españolas, como los relacionados con las tarjetas black o la falta de transparencia en la emisión de preferentes. Todos estos ejemplos evidencian la influencia que algunas personas pueden tener sobre el bienestar social y es interesante conocer las razones por las que algunos individuos se centran en su éxito individual (egosystem), mientras que otros se preocupan por procurar el bienestar a largo plazo de sus negocios y las comunidades de personas que los integran (ecosystem).

Por egosystem se entiende un conjunto de motivaciones basadas en uno mismo, en asegurar la satisfacción de las necesidades y deseos propios, donde la preocupación por el bienestar de los demás es muy baja o inexistente, y si la hay, es porque se piensa que se le puede sacar partido. Frente a esta forma de entender las relaciones entre las personas, el concepto ecosystem reconoce la existencia de gente generosa, con empatía, que se preocupa por el bienestar de los demás y deja de lado el egoísmo implícito de la concepción anterior (Crocker y Canevello, 2012).

\section{Estilos de liderazgo emergentes o contemporáneos y sus consecuencias}

Este cambio tiene también su efecto en la forma de liderar. Según Sendjaya y otros (2008), no es ninguna sorpresa el auge que ha experimentado en los últimos años la investigación sobre estilos de liderazgo cargados de valores, bajo diferentes fórmulas o términos, como pueden ser el liderazgo auténtico, el liderazgo ético, el liderazgo espiritual o el liderazgo servicial, por citar solamente algunos de ellos. Entre los trabajos recientes, cabe destacar la propuesta de Anderson y Sun (2017), quienes examinan de forma conjunta y detallada diferentes estilos que están recibiendo especial atención por parte de la literatura académica: ideológico, pragmático, servicial, auténtico, ético, espiritual, integrador, compartido, además del carismático, transformacional y transaccional. Teniendo en cuenta el interés actual de estos estilos y su juventud relativa, se puede hablar de un conjunto de estilos de liderazgo emergentes o contemporáneos.

La figura 1 pone de relieve la creciente atención que han recibido algunos de estos estilos, con un incremento, en algunos casos exponencial, de las publicaciones relacionadas con los mismos. 
Figura 1. Evolución del número de artículos académicos relacionados con algunos estilos de liderazgo emergentes o contemporáneos

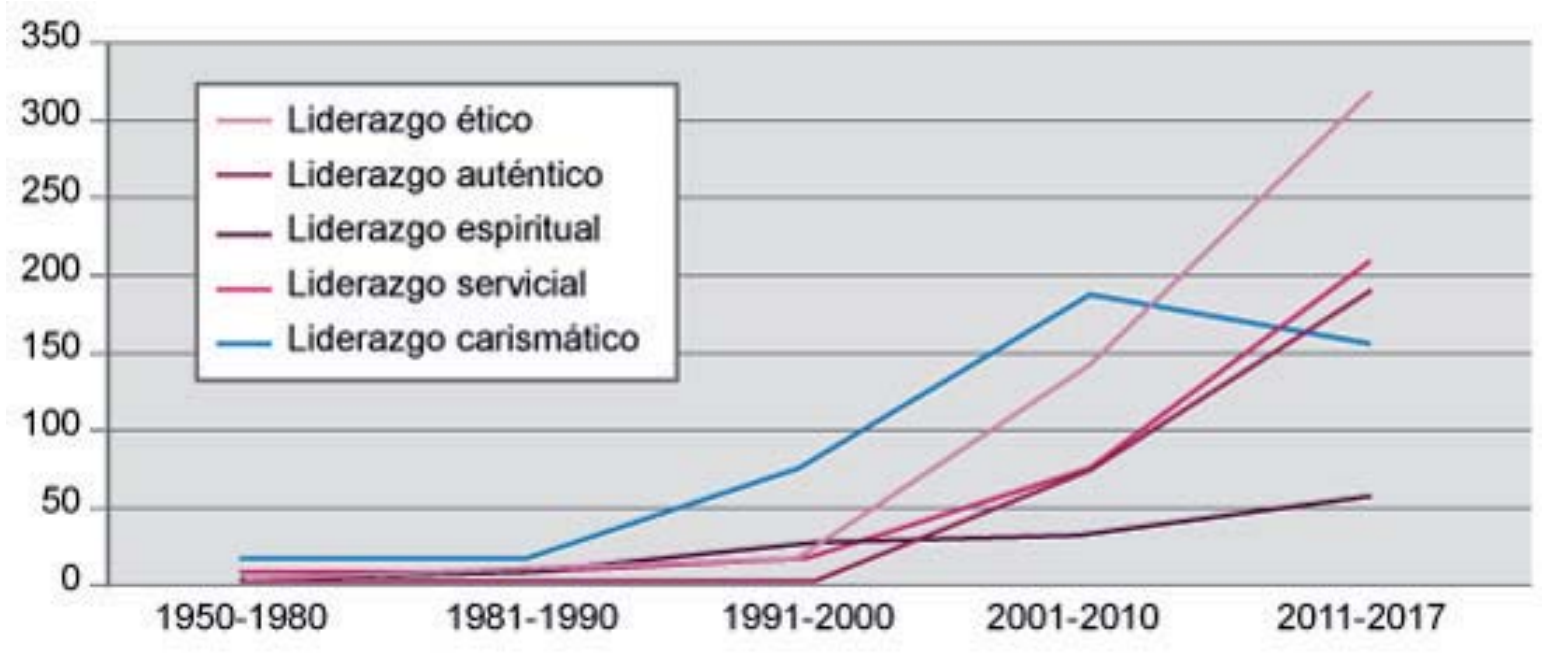

Fuente: elaboración propia a partir de Business Source Premier.

A continuación se introducen algunos estilos de liderazgo contemporáneos y se esbozan algunas de sus características y consecuencias.

El líder ético actúa de forma apropiada y promueve tal conducta en sus seguidores a través de la comunicación y la participación en la toma de decisiones (Brown y otros, 2005). En esencia, un líder ético es una persona justa, honesta y de confianza. Asimismo, actúa de acuerdo con lo que predica y es visto por los miembros de la organización como un ejemplo a seguir. Por último, utiliza la ética en su forma de dirigir y establece recompensas para que sus seguidores mantengan también una conducta ética.

Avolio y otros (2004) definen a los líderes auténticos como personas totalmente conscientes de su forma de pensar, que se comportan y son percibidas como conocedores de sus propios valores, conocimientos y fortalezas y también de las de los demás, y son reconocidas por su marcado carácter moral para actuar en beneficio del resto. Estas personas tienen confianza en sí mismas y son positivas, optimistas y flexibles. Shamir y Eilam (2005) consideran que los líderes auténticos tienen cuatro características: (1) son fieles a sí mismos, por lo que no intentan complacer a los demás sin más; (2) están motivados por convicciones personales, en lugar de estar centrados en alcanzar estatus, honores u otros beneficios personales; (3) son originales, no copian de otras personas y, por tanto, guían desde su propio punto de vista; (4) sus acciones están basadas en sus valores y convicciones personales.

El líder espiritual es definido por Fry (2003) como aquel que logra motivar e inspirar a los trabajadores a través de una visión transcendente y una cultura basada en valores altruistas para generar trabajadores motivados, comprometidos 
y productivos. El líder espiritual es aquel que muestra valores espirituales como la integridad, la honestidad, compasión, respeto y humilidad, y demuestra que se puede confiar en él o ella (Reave, 2005, pág. 663).

El líder servicial es aquel cuya prioridad es servir y satisfacer las necesidades de los demás (Greanleaf, 1970) y ayudar a las personas a desarrollarse, a esforzarse y a crecer. De acuerdo con Patterson (2003), el líder servicial es, entre otras cosas, altruista, humilde, de confianza, visionario y motivador. Estas personas también muestran una vocación altruista, son persuasivos y con coraje, demuestran capacidad de perdonar, son humildes y auténticos, se comportan de forma ética y con responsabilidad, y crean valor para la comunidad que les rodea (Anderson y Sun, 2017).

Los líderes carismáticos son capaces de transmitir la misión y la visión de la organización de forma convincente a los empleados, de forma que crean en un futuro mejor. A su vez, contribuyen a generar altos niveles de iniciativa y construir fuertes vínculos emocionales entre sus seguidores (Hugues y otros, 2009).

Numerosos trabajos demuestran consecuencias positivas de estos tipos de liderazgo, ya sea a nivel individual, grupal u organizativo. Por ejemplo, si observamos exclusivamente el liderazgo servicial, se ha demostrado la relación con mayores niveles de satisfacción de los empleados, mayor grado de apoyo percibido, menor intención de cambiar de compañía, menor nivel de estrés, mayor compromiso y confianza en la organización y sus supervisores, mayor efectividad en el desempeño de sus tareas, etc. (Van Dierendonck, 2011). Si nos referimos al liderazgo ético, se han encontrado relaciones positivas con la satisfacción de los empleados, su compromiso con la organización, mejora del desempeño, engagement e identificación con la organización (Bedi y otros, 2016), entre otros aspectos.

Todas estas variables resultan fundamentales para la supervivencia de muchas organizaciones y también lo son la creatividad y la capacidad para innovar. Estudios precedentes demuestran que estos estilos de liderazgo emergentes promueven estos aspectos en las empresas, lo que mejora su competitividad.

\section{Dificultades encontradas en su estudio y alternativas}

El estudio de estos estilos de liderazgo y sus consecuencias para la organización presenta algunas dificultades o problemas.

Como se ha explicado en líneas anteriores, estos estilos se miden mediante el uso de diferentes dimensiones: altruismo, humildad, conducta ética, etc. Se trata, por tanto, de estilos cuya amplia naturaleza lleva a que, en ocasiones, el análisis de sus consecuencias no resulte fácil. Por ejemplo, al encontrar que los líderes serviciales promueven la creatividad, innovación o mejoran el desempeño de los 
trabajadores, ¿qué variables son las que más influyen? ¿Se podrían compensar los efectos entre las mismas? Utilizar una taxonomía completa de liderazgo puede dificultar la interpretación de los resultados y, por tanto, llevar a decisiones erróneas.

Por otra parte, muchos de estos estilos presentan algún tipo de solapamiento o redundancia en su definición. Como se ha señalado, el altruismo está presente de forma explícita o implícita en la definición de estilos como el servicial, el espiritual o el ético. Y esto es algo que no solo pasa con esta dimensión, sino también con otras.

Para superar estas dificultades o problemas, algunos autores sugieren centrar el estudio del liderazgo en aspectos concretos del mismo, como, por ejemplo, el comportamiento o los rasgos del líder en contextos específos (Yukl, 2010). En los últimos años, algunos trabajos están siguiendo este enfoque. Por ejemplo, Sendjaya y Pekerti (2010) examinan diferentes dimensiones (por separado) del liderazgo servicial y su impacto sobre la confianza de los trabajadores en el líder. Otros trabajos empíricos más recientes se centran en el análisis de las consecuencias que tiene una de las dimensiones de estos estilos, concretamente el altruismo del líder, sobre el desempeño organizativo (Mallén y otros, 2015) o sobre la innovación (Domínguez-Escrig y otros, 2016).

Algunos de los comportamientos o actitudes específicas de los líderes sobre los que se podría profundizar son el altruismo, la capacidad de perdonar, la humildad, el empoderamiento, el comportamiento responsable o la orientación hacia la sostenibilidad del líder, entre otros. A continuación se explica brevemente en qué consisten estos comportamientos.

El comportamiento altruista de los líderes ha sido poco estudiado, a pesar de haber sido destacado como una interesante línea de investigación (Dinh y otros, 2014). Se trata de un tipo de comportamiento prosocial que busca ayudar a los demás sin estar pendiente de las consecuencias personales que pueda acarrear, aunque estas puedan ser negativas.

La capacidad para perdonar implica dejar pasar tanto los errores ajenos como los propios con la finalidad de aprender de los mismos (Caldwell y Dixon, 2010). Tener una orientación positiva hacia los errores, fallos y ofensas que se han cometido en el lugar de trabajo permitirá que, potencialmente, una situación de sufrimiento se convierta en una experiencia enriquecedora (Davidhizar y Laurent, 2000).

La humildad se podría definir como la disposición a intentar autoevaluarse de forma precisa y a ser conscientes del hecho de que nadie es perfecto (Templeton, 1997). Las personas humildes son vistas como comprensivas, dispuestas a admitir sus propios errores y a aprender de ellos. Owens y otros (2013), además de lo anterior, incluyen en la definición de humildad la consideración de las contribuciones y puntos fuertes de los demás, así como la apertura a nuevas ideas y el feedback. 
Aunque se considera que la humildad de los líderes es una de las claves para valorar su efectividad, los estudios empíricos sobre humildad en los líderes son todavía escasos.

El empoderamiento se define como el proceso por el que los líderes o directivos comparten el poder con sus empleados, los motivan para que realicen sus tareas sintiendo que son autónomos y eficaces. Estos líderes subrayan la importancia del trabajo realizado por los trabajadores, muestran confianza en las capacidades de los empleados, facilitan su participación en la toma de decisiones, promueven el autoliderazgo, comparten información, establecen objetivos significativos, inspiran y ofrecen una visión excitante del futuro, etc.

El comportamiento responsable se puede definir como la medida en que un individuo subordina voluntariamente sus intereses personales y piensa en el bienestar a largo plazo de sus subordinadoes y de la sociedad en general. Por otro lado, la orientación hacia la sostenibilidad es un concepto muy próximo al que se ha descrito para el comportamiento responsable, y se entiende como la preocupación por el bienestar de la sociedad y del medioambiente, para lo que se adopta una visión de largo plazo de tales cuestiones (Eisenbeiss, 2012). En este sentido, algunos autores (por ejemplo, Karns, 2011) enfatizan la necesidad de promover este tipo de comportamientos para fortalecer una economía que incorpore una visión más humana y sostenible para las generaciones futuras.

\section{Conclusiones}

Varios factores han contribuido en los últimos años a la aparición de nuevas formas de liderar las organizaciones y han adquirido cada vez mayor protagonismo estilos de liderazgo en los que predominan los valores humanos y la preocupación e interés por los demás. Estos nuevos estilos han recibido diferentes nombres y definiciones, pero en la mayoría de ellos se encuentran características comunes, como la humildad, el altruismo o la capacidad de perdonar.

Se ha estudiado qué efectos tienen estos estilos de liderazgo emergentes sobre variables como la innovación, la creatividad, el desempeño, la confianza o el compromiso de los empleados con la organización y sus supervisores, y los resultados son alentadores. Sin embargo, a la hora de trasladar a la empresa qué conductas se deben promover entre las personas que las lideran, parece razonable profundizar en el análisis del efecto de conductas o comportamientos concretos como los mencionados, ya que hasta la fecha la investigación es relativamente escasa. Un buen comienzo puede ser centrar la investigación en aquellas dimensiones o aspectos que se repiten en los estilos de liderazgo que están adquiriendo relevancia en la actualidad. 
Los resultados de estas investigaciones pueden suponer avances para la literatura académica, pero también para las empresas. Desde un punto de vista aplicado, las organizaciones pueden llevar a cabo procesos de selección de personal en los que se valoren comportamientos o conductas presentes en los estilos de liderazgo emergentes que se han presentado. Muchas de estas conductas pueden promoverse también a través de la formación a los empleados y serán más fáciles de transmitir si los nuevos líderes dan ejemplo y se comportan de igual modo. Aún se podría dar un paso más y tratar de inculcar estos comportamientos a estudiantes universitarios, como futuros líderes de nuestras empresas, a través de casos de estudio. A la vista del impacto de algunas de estas conductas o comportamientos sobre la innovación o el desempeño, las empresas también se podrían plantear valorarlas al evaluar a sus trabajadores y ser un factor más a tener en cuenta en sus sistemas retributivos o en sus políticas de promoción.

\section{Referencias bibliográficas}

ANDERSON, M. H.; SUN, P. I. (2017). «Reviewing leadership styles: Overlaps and the need for a new 'full-range' theory». International Journal of Management Reviews. Vol. 19, n. ${ }^{\circ}$ 1, págs. 76-96.

AVOLIO, B. J.; GARDNER, W. L.; WALUMBWA, F. O.; LUTHANS, F.; MAY, D. R. (2004). «Unlocking the mask: A look at the process by which authentic leaders impact follower attitudes and behaviors». The Leadership Quarterly. Vol. 15, n. ${ }^{\circ}$ 6, págs. 801-823.

BEDI, A.; ALPASLAN, C. M.; GREEN, S. (2016). "A meta-analytic review of ethical leadership outcomes and moderators». Journal of Business Ethics. Vol. 139, n. ${ }^{\circ}$, págs. 517-536.

BROWN, M. I.; TREVIÑO, L. K.; HARRISON, D. A. (2005). «Ethical leadership: A social learning perspective for construct development and testing». Organizational behavior and human decision processes. Vol. 97, n. 2, págs. 117-134.

CALDWELL, C.; DIXON, R. D. (2010). «Love, forgiveness, and trust: Critical values of the modern leader». Journal of Business Ethics. Vol. 93, n. ${ }^{\circ 1}$, págs. 91-101. CROCKER, J.; CANEVELLO, A. (2012). «Egosystem and ecosystem: Motivational perspectives on caregiving». En: BROWN, S.; BROWN, M.; Penner, L. A. (eds.). Moving beyond self-interest: Perspectives from evolutionary biology, neuroscience, and the social sciences (págs. 211-223). Nueva York, NY: Oxford University Press.

DAVIDHIZAR, R. I.; LAURENT, C. R. (2000). «The art of forgiveness». Hospital materiel management quarterly. Vol. 21, n. ${ }^{\circ}$ 3, págs. 48. 
DE CIERI, H.; HOLMES, B.; ABBOTT, J.; PETTIT, T. (2005). «Achievements and challenges for work/life balanç strategies in Australian organizations». The International Journal of Human Resource Management. Vol. 16, n.¹, págs. 90-103. DINH, J. I. ; LORD, R. G. ; GARDNER, W. L.; MEUSER, J. D.; LIDEN, R. C.; HU, J. (2014). «Leadership theory and research in the new millennium: Current theoretical trends and changing perspectives». The Leadership Quarterly. Vol. 25, n. 1, págs. 36-62. DOMíNGUEZ-ESCRIG, I.; MALLÉN, F. F.; CHIVA, R.; LAPIEDRA, R. (2016). «How does altruistic leader behavior foster radical innovation? The mediating effect of organizational learning capability». Leadership \& Organization Development Journal. Vol. 37, n. ${ }^{\circ}$ 8, págs. 1056-1082.

EISENBEISS, S. A. (2012). «Re-thinking ethical leadership: An interdisciplinary integrative approach». The Leadership Quarterly. Vol. 23, n. ${ }^{\circ}$ 5, págs. 791-808.

FRY, L. W. (2003). «Toward a theory of spiritual leadership». The Leadership Quarterly. Vol. 14, n. 6 , págs. 693-727.

GREENLEAF, R. (1970). The servant as leader. Indianapolis, IN: Robert K. Greenleaf Center.

HUGHES, R. L.; GINNETT, R. C.; CURPHY, G. J. (2009). Leadership: Enhancing the lessons of experience (6. ${ }^{a}$ ed.). Nueva York, NY: McGraw-Hill/Irwin.

KARNS, G. L. (2011). "Stewardship: a new vision for the purpose of business». Corporate Governance: The International Journal of Business Insociety. Vol. 11, n. ${ }^{\circ}$, págs. 337-347.

MALLÉN, F.; CHIVA, R.; ALEGRE, J.; GUINOT, J. (2015). «Are altruistic leaders worthy? The role of organizational learning capability». International Journal of Manpower. Vol. 36, n. ${ }^{\circ}$ 3, págs. 271-295.

OWENS, B. P.; JOHNSON, M. D.; MITCHELL, T. R. (2013). «Expressed humility in organizations: Implications for performance, teams, and leadership». Organization Science. Vol. 24, n. ${ }^{\circ}$, págs. 1517-1538.

PATTERSON, K. A. (2003). Servant leadership: A theoretical model. Virginia Beach, VA: Regent University.

REAVE, L. (2005). "Spiritual values and practices related to leadership effectiveness». The Leadership Quarterly. Vol. 16, n. ${ }^{\circ}$ 5, págs. 655-687.

SENDJAYA, S.; CARRALLS, J. C.; SANTORA, J. C. (2008). «Defining and measuring servant leadership behaviour inorganizations». Journal of Management Studies. Vol. 45, n.² 2, págs. 402-424.

SENDJAYA, S.; PEKERTI, A. (2010). «Servant leadership as antecedent of trust inorganizations». Leadership \& Organization Development Journal. Vol. 31, n. 7, págs. 643-663.

SHAMIR, B.; EILAM, G. (2005). «"What's your story?” A life-stories approach to authentic leadership development». The Leadership Quarterly. Vol. 16, n. ${ }^{\circ}$, págs. 395-417. 
TEMPLETON, J. M. (1997). Worldwide Laws of Life. Philadelphia: Templeton Foundation Press.

VAN DIERENDONCK, D. (2011). «Servant leadership: A review and synthesis». Journal of Management. Vol. 37, n. 4, págs. 1228-1261.

Yukl, G. (2010). Leadership in organizations (7. ${ }^{a}$ ed. internacional). Londres: Prentice Hall.

ZHU, W.; CHEW, I. K.; SPANGLER, W. D. (2005). «CEO transformational leadership and organizational outcomes: The mediating role of human-capital-enhancing human resource management». The Leadership Quarterly. Vol. 16, n. ${ }^{\circ 1}$, págs. 39-52.

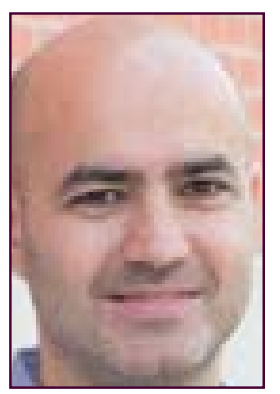

\section{Francisco Fermín Mallén Broch fmallen@uji.es Universitat Jaume I}

Doctor en Gestión Empresarial por la Universitat Jaume I (UJI), máster universitario en Psicología del Trabajo, de las Organizaciones y en Recursos Humanos (UJI), máster en Investigación de Mercados y Sistemas de Información (UNED), licenciado en Ciencias y Técnicas Estadísticas (Universidad de Valencia) y licenciado en Administración y Dirección de Empresas (UJI). Actualmente, es profesor en el Departamento de Administración de Empresas y Marketing de la Universitat Jaume I (Castellón), donde ha impartido asignaturas relacionadas con dirección de recursos humanos, sistemas de información y dirección estratégica, entre otras. Algunos de sus trabajos han sido publicados en revistas como Journal of Business Ethics, International Journal of Manpower o Leaderhip and Organizational Development Journal. Su área de investigación está relacionada con el liderazgo, la innovación y el aprendizaje organizativo. 


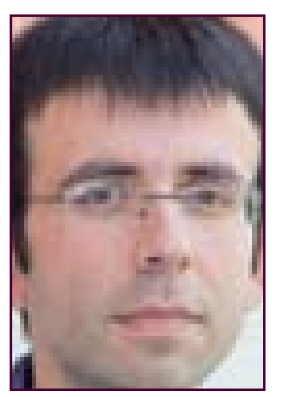

\section{Fmilio Dominguez Fscrig edomingu@uji.es Universitat Jaume I}

Máster en Marketing e Investigación de Mercados (UJI) y licenciado en Publicidad y Relaciones Públicas (UJI). Ha sido professor asociado del Departamento de Administración de Empresas y Marketing de la Universitat Jaume I (Castellón), donde ha impartido asignaturas relacionadas con la dirección de recursos humanos. Actualmente, es investigador en formación en esta misma universidad y elabora una tesis doctoral que analiza los antecedentes de la innovación radical en las organizaciones. Su investigación aborda temas como el empoderamiento, la capacidad para perdonar, el altruismo o el aprendizaje. Ha publicado en revistas como Leadership and Organizational Development Journal.

Los textos publicados en esta revista están sujetas -salvo que se indique el contrario- a una licencia de Reconocimiento 3.0 España de Creative Commons. Podéis copiarlos, distribuirlos, comunicarlos públicamente y hacer obras derivadas siempre que reconozcáis los créditos de las obras (autoría, nombre de la revista, institución editora) de la manera especificada por los autores o por la revista. La licencia completa se puede consultar en http://creativecommons.org/licenses/by/3.0/es/deed.ca.

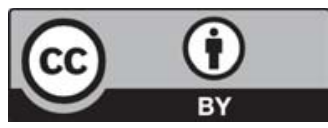

